\title{
Efectos de la musicoterapia en el nivel de intensidad del dolor post quirúrgico en pacientes pediátricos en un hospital de EsSALUD.
}

\author{
Bernice Yemira Ortiz Monroy ${ }^{1, a}$, Doris Velásquez Carranza ${ }^{21, b, 2}$
}

\section{RESUMEN}

Objetivos: determinar los efectos de la musicoterapia, como tratamiento complementario, en el nivel de intensidad del dolor postquirúrgico en pacientes pediátricos en el servicio de Neurocirugía del Hospital Nacional Guillermo Almenara Irigoyen. Material y Métodos: estudio cuasi experimental. Muestra: 40 pacientes pediátricos de 5 a 17 años, 20 pacientes del grupo experimental que recibieron sesión de musicoterapia clásica por 30 minutos y 20 pacientes grupo control quienes no recibieron sesión de musicoterapia, en ambos grupos se utilizaron escalas CHEOPS (de 5 a 7 años), facial (8 a 17 años). Resultados: el puntaje del dolor en el grupo experimental de 8 a 17 años a las 24 horas post operados fue de $5 \pm 1,9$ (moderado) y posterior a la musicoterapia, el puntaje del dolor fue de 3,2 $\pm 1,6$ (moderado), encontrándose una reducción del dolor en 1,8 puntos, estadísticamente significativa $\mathrm{P}=0,000<0,05 \mathrm{y}$ en niños de 5 a 7 años fue de $9,6 \pm 1,9$ (moderado); después de 30 minutos con musicoterapia el puntaje del dolor fue de 7,6 $\pm 1,8$ (leve). Se encontró una reducción del dolor en 2 puntos, estadísticamente significativa $\mathrm{P}=0,000<0,05$; en el grupo control en ambos grupos no hubo cambios significativos. Conclusiones: la musicoterapia disminuye el nivel de intensidad del dolor postquirúrgico en pacientes pediátricos en el servicio de Neurocirugía del Hospital Nacional Guillermo Almenara Irigoyen.

PALABRAS CLAVE: Musicoterapia, intensidad del dolor, cuasi experimento.

\section{SUMMARY}

\section{Effects of music therapy in intensity level post surgical pain in pediatric patients in EsSALUD hospital.}

Objectives: delimit the effects of music therapy, as complementary treatment, on the level of intensity of postoperative pain in pediatric patients in the Neurosurgery Department of the Hospital Guillermo Almenara Irigoyen. Material and Methods: design quasi-experimental, sample: 40 pediatric patients of 5-17 years old, 20 patients of experimental group received classical music therapy session for 30 minutes and 20 patients of control group not received music therapy session, both groups used CHEOPS scales (5-7 years), facial (8-17 years). Results: the pain score in the experimental group of 8-17 years after 24 hours of post-surgery was $5 \pm 1.9$ (moderate) and after music therapy, the pain score was $3.2 \pm 1,6$ (moderate). It was found a reduction in pain of 1,8 points statistically significant $\mathrm{P}=0.000<0,05$; and children of 5-7 years was $9.6 \pm 1.9$ (moderate); after 30 minutes with music therapy, the pain score was $7.6 \pm 1.8$ (mild). In the control group it was a found a reduction in pain of 2 points statistically significant $\mathrm{P}=0.000<0.05$ in both groups there were no significant changes. Conclusions: Music therapy decreases the level of intensity of postoperative pain in pediatric patients in the Neurosurgery Department of National Hospital Guillermo Almenara Irigoyen.

KEYWORDS: music therapy, pain intensity, quasi experiment.

Enfermera asistencial del Hospital Guillermo Almenara Irigoyen. Lima-Perú.

Docente Asociada de la Facultad de Enfermería de la Universidad Peruana Cayetano Heredia.

Licenciada en enfermería;

Magister de Medicina. 


\section{INTRODUCCIÓN}

El dolor constituye una experiencia universal que afecta a toda la población en algún momento de su vida (1). La cuantificación del dolor es difícil en el adulto y es mucho más difícil cuantificarla en niños y adolescentes, por lo que aliviarlo es una prioridad dentro del cuidado enfermero. La musicoterapia, como una terapia complementaria a la farmacológica, representa una alternativa de cuidado para el manejo del dolor, por lo que surge la necesidad de investigar cuál es su efecto en el alivio del dolor y así brindar una terapia adicional que complemente su recuperación.

En el cuidado del paciente pediátrico, la principal preocupación de la enfermera es el bienestar del niño, del adolescente y también de su familia; por esa razón, la enfermera debe tener conocimientos de las reacciones al dolor en los diferentes periodos del desarrollo (2). La Asociación Internacional para el Estudio del Dolor (IASP) define al dolor como "una experiencia sensorial y emocional desagradable, asociada a una lesión real o potencial, o descrita en términos de dicho daño"; como tal, causa malestar en toda persona y con mucha mayor razón en aquellos que sufren de intervenciones quirúrgicas, pues es sabido que todo tipo de cirugía va a causar dolor en el paciente, ya sea de intensidad leve, moderada o severa (3).

El dolor en el paciente pediátrico es muy frecuente en el periodo post quirúrgico inmediato y mediato; ya sea de una cirugía mayor o menor el dolor se presentará por la injuria causada en su organismo (3). En los últimos 15 años, los mitos relacionados con la falta de percepción dolorosa en el paciente pediátrico han sido sustituidos por investigaciones epidemiológicas. Sin embargo, aún no se ha establecido una correlación adecuada entre los nuevos conceptos sobre la percepción dolorosa, la adecuada evaluación y manejo del problema (4).

En el periodo post quirúrgico, el dolor varía de intensidad dependiendo del tipo de cirugía, del tiempo transcurrido después de ésta y del umbral de tolerancia del paciente. Adicionalmente, en los servicios de Neurocirugía, los pacientes pediátricos son sometidos a múltiples procedimientos invasivos y no invasivos, permanecen aislados, en algunos casos, con sujeción mecánica, lo que genera además, estrés e incertidumbre. Un inadecuado manejo del dolor en la fase aguda repercute en la morbilidad; ya que podría ocasionarse que las respuestas neuroendocrinas, metabólicas y emocionales que se originan en el organismo como consecuencia del estrés que produce una situación dolorosa, resultan perjudiciales para la recuperación del paciente (5). El tratamiento no farmacológico para el alivio del dolor es una técnica que se está utilizando cada vez más en las áreas o unidades de dolor para el alivio del mismo. Dentro de estos tipos de terapia complementaria tenemos la musicoterapia, que viene siendo aplicado en otros países por especialistas en música (6).

El objetivo del presente trabajo fue determinar los efectos de la musicoterapia como terapia complementaria en el nivel de intensidad del dolor postquirúrgico en pacientes pediátricos de 5 a 17 años, en el servicio de Neurocirugía del Hospital Nacional Guillermo Almenara Irigoyen de setiembre del 2014 hasta abril del 2015.

\section{MATERIAL Y MÉTODOS}

\section{DISEÑO DEL ESTUDIO}

El estudio es de diseño cuasi experimental :

$\begin{array}{llll}\text { GE } & \mathrm{O} 1 & \mathrm{X} & \mathrm{O} 2 \\ \text { GC } & \mathrm{O} 3 & & \mathrm{O} 4\end{array}$

GE : Grupo experimental.

GC : Grupo control.

$\mathrm{X}$ : Intervención con la musicoterapia como terapia complementaria.

O1: Observación a las 24 horas de post operado.

$\mathrm{O} 2$ : Observación después 30 minutos de la musicoterapia.

O3: Observación a las 24 horas de post operado.

O4: Observación a las 24.30 horas de post operado.

El estudio de investigación se realizó durante 8 meses, desde setiembre del 2014 hasta abril del 2015, en el, en el, en el Area de cuidados intensivos y cuidados intermedios del Servicio de Neurocirugía del Hospital de IV nivel de atención Guillermo Almenara Irigoyen .

La población estuvo conformada por pacientes pediátricos con edades entre los 5 y 17 años de ambos sexos, post operados de cirugías vertebrales, derivaciones ventriculares, evacuación de hematomas y/o extirpación de lesiones. Según información de la Unidad de Estadística del Hospital Nacional Guillermo Almenara Irigoyen, hay un promedio mensual de nueve pacientes operados en este rango de edad y en el 2013 fueron 120 pacientes (6). El diseño muestral corresponde a un muestreo no probabilístico por saturación. 
Para el cálculo de la muestra, como no se conocía la proporción de pacientes que presentaron algún nivel de intensidad del dolor antes y después de la aplicación de música, se tomó una prueba piloto realizada en el Hospital Nacional Guillermo Almenara Irigoyen, a un total de 20 pacientes de 5 a 17 años, donde 10 pacientes son del grupo experimental y 10 del grupo control, obteniéndose las siguientes estimaciones:

Se obtuvo como resultado 20 pacientes por grupo, que fueron estratificados según edad: 5 a 7 años y 8 a 17 años.

\section{Criterios de inclusión:}

Paciente despierto, lúcido y afebril.

Paciente que ventile espontáneamente.

Que tengan el consentimiento informado de sus padres $\mathrm{y} / \mathrm{o}$ asentimiento informado.

Post operado inmediato ( 24 horas post cirugía).

Haber recibido su analgésico 8 horas antes.

Edad de 5 a 17 años.

\section{Criterios de exclusión:}

Paciente con diagnóstico de hipoacusia, sordera.

Para medir la intensidad del dolor en el grupo de 8 a 17 años se utilizó, la técnica de la entrevista y como instrumento la escala facial, y en el grupo de 5 a 7 años se aplicó la técnica de la observación y como instrumento la escala CHEOPS.

La escala facial fue usada en los pacientes pediátricos entre de 8 a 17 años, en el grupo experimental y grupo control. Cuando el paciente expresa su dolor este dato fue registrado en el instrumento esta valoración se realizó a las 24 horas posterior a la intervención quirúrgica y 24 y media horas en ambos grupos, con la diferencia que en el grupo experimental en el lapso de los 30 minutos se aplicó la musicoterapia.
Escala de CHEOPS la cual cuenta con validación, usada en el paciente pediátrico de 5 a 7 años, donde se recoge datos generales e incluye: fecha, edad, sexo, día y hora de la cirugía; incluye, además, un cuadro que valora el dolor según conductas: expresión facial, verbalización, actitud corporal, deseo de tocar la herida, movimiento de extremidades inferiores, gritos y llantos, que tiene el niño. Teniendo como resultado de la medición los siguientes valores: 4 puntos sin dolor, 5-8 puntos dolor leve, 9-11 puntos dolor moderado y 12-13 puntos dolor severo.

El instrumento fue aplicado a las 24 horas posterior a la intervención quirúrgica y 24.30 horas en ambos grupos, con la diferencia que en el grupo experimental en el lapso de los 30 minutos se aplicó la musicoterapia.

Estos instrumentos fueron validados a través de juicio de 10 expertos en el tema.

\section{RESULTADOS}

Los resultados del presente estudio se muestran en las tablas $1,2,3$ y 4 .

\section{DISCUSION}

En la tabla 1 y 2, se puede observar que después de la intervención con musicoterapia, en el grupo experimental versus el grupo control, en pacientes de 8 a 17 años se redujo la intensidad del dolor en un 1,6 puntos $(\mathrm{p}=0,001)$ y en pacientes de 5 a 7 años en 1,8 puntos $(\mathrm{p}=0.002)$, ambas diferencias resultaron estadísticamente significativas.

Estos resultados coinciden con el estudio realizado por Hatmen y Lira, sobre los efectos de la música en los niños después de la cirugía cardiaca donde evaluaron el dolor y funciones vitales antes y después de la sesión de musicoterapia, y en el grupo control fue aplicado un

Tabla 1. Comparación de la intensidad del dolor después de la intervención en el grupo experimental vs el grupo control en pacientes post operados de 8 a 17 años en el Hospital Nacional Guillermo Almenara Irigoyen setiembre 2014 - abril 2015 Lima - Perú

\begin{tabular}{cccccc}
\hline GRUPO & Media & $\mathrm{N}$ & Desviación estándar & $\mathrm{T}+$ & $\mathrm{P}$ \\
\hline $\begin{array}{c}\text { Experimental } \\
\text { Diferencia } \\
\text { Control }\end{array}$ & 1,8 & 10 & 0,6 & 3,8 & $0,001^{*}$ \\
\hline
\end{tabular}

+ T student $* \mathrm{P}<0,05$ estadísticamente significativo 
Tabla 2.Comparación de la intensidad del dolor después de la intervención en el grupo experimental vs grupo control en pacientes post operados de 5 a 7 años en el Hospital

Nacional Guillermo Almenara Irigoyen setiembre 2014 - abril 2015 Lima - Perú

\begin{tabular}{|c|c|c|c|c|c|}
\hline GRUPO & Media & $\mathrm{N}$ & Desviación estándar & $\mathrm{T}+$ & $\mathrm{P}$ \\
\hline Experimental & 2,0 & 10 & 1,0 & & \\
\hline Diferencia & & & & 3,5 & $0,002 *$ \\
\hline Control & 0,2 & 10 & 1,2 & & \\
\hline
\end{tabular}

$+\mathrm{T}$ student $* \mathrm{P}<0,05$ estadísticamente significativo

Tabla 3. Comparación de medias en el nivel de intensidad del dolor del grupo experimental en pacientes de 8 a 17 años Hospital Nacional Guillermo Almenara Irigoyen setiembre 2014 - abril 2015 Lima - Perú.

\begin{tabular}{|c|c|c|c|c|c|}
\hline & Media & $\mathrm{N}$ & Desviación estándar & $\mathrm{T}+$ & $\mathrm{P}$ \\
\hline \multirow[t]{2}{*}{ A las 24 horas post operados } & 5 & 10 & 1,9 & 9 & $0,000 *$ \\
\hline & Media & $\mathrm{N}$ & Desviación estándar & $\mathrm{T}$ & $\mathrm{P}$ \\
\hline A las 24 horas post operados & 4,2 & 10 & 1,4 & 0,5 & 0,55 \\
\hline $\begin{array}{l}\text { Después de } 30 \text { minutos sin } \\
\text { Musicoterapia }\end{array}$ & 4 & 10 & 1,3 & & \\
\hline Después de 30 minutos con musicoterapia & 3,2 & 10 & 1,6 & & \\
\hline
\end{tabular}

Tabla 4. Comparación de medias en el nivel de intensidad del dolor del grupo experimental en pacientes de 5 a 7 años Hospital Nacional Guillermo Almenara Irigoyen setiembre 2014 - abril 2015 Lima - Perú.

\begin{tabular}{lccccc}
\hline & Media & $\mathrm{N}$ & Desviación estándar & $\mathrm{T}$ & $\mathrm{P}$ \\
\hline A las 24 horas post operados & 9,6 & 10 & 1,9 & 6 & $0,000^{*}$ \\
& Media & $\mathrm{N}$ & Desviación estándar & $\mathrm{T}$ & $\mathrm{P}$ \\
$\begin{array}{l}\text { A las 24 horas post operados } \\
\begin{array}{l}\text { Después de 30 minutos sin la } \\
\text { aplicación de la musicoterapia }\end{array}\end{array}$ & 9,3 & 10 & 0,6 & 0,51 & 0,61 \\
\begin{tabular}{l} 
Después de 30 minutos con musicoterapia \\
\hline
\end{tabular} & 9,1 & 10 & 1,5 & & \\
\hline
\end{tabular}

+ T student $* \mathrm{P}<0,05$ estadísticamente significativo

placebo un $\mathrm{CD}$ sin música ambos por 30 minutos, en dichos resultados del estudio se observaron diferencias estadísticamente significativas en los parámetros de dolor, frecuencia cardiaca y frecuencia respiratoria $(\mathrm{p}<0,001$, $\mathrm{p}$ $=0,04$ y $\mathrm{p}=0,02$, respectivamente) $(7)$.

La utilización de música puede reducir el estrés y hasta calmar dolores agudos, incrementando sentimientos de control y logrando concentrarse en situaciones positivas que alejan el dolor, emitiendo sonidos que regulan el equilibrio homeostático, el cual estimula las glándulas de secreción interna a través de la estimulación al hipotálamo, el cual su vez liberan dos sustancias encefálicas, que son: beta endorfinas y dinorfinas, que son sustancias producto de degradación de grandes moléculas proteicas que forman parte del sistema del control del dolor (analgésica) del cerebro, lo que puede disminuir en su totalidad o casi por completo muchas señales del dolor, así también actúa a nivel del sistema parasimpático regulando el trabajo coordinado del sistema nervioso autónomo $(8,9)$. 
En la tabla 3, se puede observar que el puntaje del dolor en el grupo experimental de 8 a 17 años de edad, a las 24 horas post operados, fue de $5 \pm 1,9$ que se interpreta como dolor moderado, en la escala facial. Después la aplicación de la música por 30 minutos, el puntaje del dolor fue de 3,2 $\pm 1,6$ encontrándose también dentro del inicio de la escala de dolor moderado. Habiendo una reducción del dolor de 1,8 puntos estadísticamente significativa $\mathrm{P}=0,000<0,05$ (tabla 3 ). Según Cépeda, en su revisión sistemática, observó que la musicoterapia aliviaba en casi un punto el dolor de los afectados y que necesitaban $1 \mathrm{mg}$ menos de morfina en comparación con los pacientes que no fueron expuestos a la música. Sin embargo, debido a que solo se observó la disminución de un punto en la escala EVA, es insuficiente la relevancia clínica de la música para el alivio del dolor en la práctica clínica, pero dado que es una terapia complementaria a la farmacológica, sí tiene relevancia ya que va a contribuir a aliviar el dolor (9).

Estos resultados coinciden con la investigación de Cépeda, donde demostró el efecto de la audición de música sobre la intensidad del dolor en pacientes post operados oncológicos (9); asimismo, los resultados coinciden con la investigación de Hatem, Lira y Matos, quienes demostraron que existe un efecto beneficioso de la música en los pacientes de post-cirugía cardíaca, a través de una serie de signos vitales (frecuencia cardíaca y respiratoria) y de la reducción del dolor (escala facial) (7).

Estos resultados también se observan en el presente estudio ya que en el grupo experimental hay una disminución del nivel de intensidad del dolor en pacientes post quirúrgico de 8 a 17 años, quizá el resultado se evidenciaría mucho más si el paciente hubiera elegido su música, ya que a esta edad el adolescente tiene su estilo musical es así que en el estudio de Hatem y Lira, dos pacientes se negaron a participar ya que tenían un gusto personal bien definido de música, lo que impidió el uso de la música clásica.

Del mismo modo, en la tabla 3, del grupo control de 8 a 17 años, donde se evaluó el dolor con la escala facial a los pacientes pediátricos a las 24 horas post cirugía, las medias no mostraron variación significativa antes y después de 30 minutos sin la aplicación de la música, teniendo una variación de 4,2 a 4 puntos, en la escala del dolor que se considera como moderado. Cabe recalcar que a ningún paciente se le retiró su terapia farmacológica prescrita para el alivio del dolor en el Hospital Guillermo Almenara Irigoyen; el medicamento habitual es el fármaco dipirona, según su peso, cada 8 horas y/o condicional, se inicia su aplicación a las 24 horas de post operado en una hora lento y diluido programado en una bomba de infusión durante el final y el inicio de la siguiente dosis (dolor episódico) (10).

En la tabla 4, se aprecia que el puntaje del dolor en el grupo experimental de 5 a 7 años, quienes fueron evaluados mediante la escala de CHEOPS y se les aplicó la música a las 24 horas post operados, fue de 9,6 $\pm 1,9$ dentro del rango moderado y después de 30 minutos con musicoterapia, el puntaje del dolor fue de 7,6 $\pm 1,8$ considerado dentro de la escala como leve, encontrándose una reducción del dolor 2 puntos estadísticamente significativa $\mathrm{P}=0,000<0,05$, observándose así un mejor resultado en niños de 5 a 7 años.

Según Rivera, en su estudio realizado, encontró que los niños entre 2 y 6 años, al ser evaluados con la escala de CHEOPS, la música clásica parece generar mayor efecto analgésico durante el procedimiento de dígito-punción (11). Según el punto de vista de Benenzon (12), la musicoterapia se fundamenta en el principio del ISO ("igual" en griego), el ISO es un conjunto de sonidos y/o fenómenos sonoros internos que nos caracteriza y nos individualiza, los niños responden mejor a la música clásica ya que su ISO universal identifica a todos los seres humanos, independientemente de sus contextos sociales, culturales, históricos y psicofisiológicos particulares, se refiere al latido cardíaco, los sonidos de inspiración y expiración o la voz de la madre en los primeros momentos del nacimiento y este ISO del niño, según su crecimiento, va a ir evolucionando según los procesos dinámicos de aprendizaje de la cultura propia (12); estos resultados coinciden con el presente trabajo de investigación ya que se pudo observar una mayor respuesta de la música clásica en niños debido quizás evoca a un entorno más favorable.

Por otro lado en la tabla 4, del grupo control de 5 a 7 años, donde se evaluó el dolor con la escala CHEOPS a las 24 horas post cirugía, las medias no mostraron variación significativa antes y después de 30 minutos sin la aplicación de la música teniendo una variación de 9,3 a 9,1 puntos, en la escala del dolor que es considerado como moderado. Cabe recalcar que ningún paciente se le retiro su terapia farmacológica prescrita para el alivio del dolor en el Hospital Guillermo Almenara Irigoyen el medicamento habitualmente de elección es el fármaco dipirona, según su peso, cada 8 horas y/o condicional, y se inicia su aplicación a las 24 horas de post operado en una hora lento y diluido programado en una bomba 
de infusión durante el final y el inicio de la siguiente dosis (dolor episódico) (7).

\section{Correspondencia:}

\section{REFERENCIAS BIBLIOGRÁFICAS:}

1. Olivares-Crespo ME, Cruzado-Rodríguez J. Evaluación psicológica del dolor. clínica y salud. 2008; 19(3):321-341.

2. Haley D. Reacción del niño y su familia a la enfermedad y a la hospitalización en enfermería pediátrica. 4ta edición. Barcelona: Mosbi-Doyma; 1993. p.550-551.

3. Moreno C, Prada D. Fisiopatología del dolor clínico.Bogota: Asociaicon Colombiana de Neurologia, 2009. (citado el 10 de enero del 2012). Disponible en: http://www.acnweb. org/guia/g3cap2.pdf.

4. Gancedo-García C, Malmierca-Sánchez D, HernándezGancedo C, Reinoso-Barbero F. Pediatría integral. Curso de formación continuada de dolor en pediatría;2008. p. 3-23. (citado el 10 de diciembre del 2011): 3-23. (citado el 10 de enero del 2012). Disponible en: http://goo.gl/6ceAED

5. Martínez J. Prevalencia del dolor post operatorio: Alteraciones fisiopatológicas y sus repercusiones. Rev Soc Esp Dolor. 2000;5:465-476.

6. Del Olmos M. Musicoterapia con bebes de 0-6 meses en cuidados intensivos pediátricos. Tesis Doctoral. Madrid, España: Universidad Autonoma de Madrid; 2009.

7. Hatem T, Lira P, Mattos S. Efeito terapéutico da música em crianças em pós-operatório de cirugia cardíaca. Jornal de Pediatría. 2006;82(3):186-192. (citado el 10 de junio del 2010) Disponible en: http://www.scielo.br/scielo. php?script $=$ sci_arttext\&pid=S0021-75572006000300006
8. Bruscia K. Definiendo Musicoterapia. España-Salamanca: Amarú Ediciones; 1997.

9. Cepeda MS, Carr DB, Lau J, Alvarez H. Music for pain relief. Cochrane Database Syst Rev. 2006(2):CD004843

10. Nabal M, Madrid F. Dolor episódico: definición, etiología y epidemiología. Rev Soc Esp Dolor. 2002; 9: 88-93.

11. Rivera G. Comparación de tres géneros musicales como método analgésico no farmacológico. Revista Peruana de Pediatría. 2008; 61: 276- 280.

12. Almanza P. La terapia musical como intervención de enfermera. Rev Enfermería Global. 2003; 2: 2-3.

\section{Correspondencia:}

Bernice Ortiz Monroy

Correo electrónico: bernice.ortiz.m@upch.pe

Fecha de Recepción: 17 de abril del 2016. Fecha de aceptación: 31 de mayo del 2016. 